\title{
Coupled cluster parametrizations of model field theories and their Bargmann-space representations
}

\author{
Document Version \\ Accepted author manuscript
}

Link to publication record in Manchester Research Explorer

\section{Citation for published version (APA):}

Bishop, RF., \& Arponen, JS. (1990). Coupled cluster parametrizations of model field theories and their Bargmannspace representations. In Y. Avishai (Ed.), Recent Progress in Many-Body Theories, Vol. 2 (pp. 193-207). Plenum Publishing Corporation. http://personalpages.manchester.ac.uk/staff/raymond.bishop/RFB_papers/[084] RPMBT_2(1990)193

\section{Published in:}

Recent Progress in Many-Body Theories, Vol. 2

\section{Citing this paper}

Please note that where the full-text provided on Manchester Research Explorer is the Author Accepted Manuscript or Proof version this may differ from the final Published version. If citing, it is advised that you check and use the publisher's definitive version.

\section{General rights}

Copyright and moral rights for the publications made accessible in the Research Explorer are retained by the authors and/or other copyright owners and it is a condition of accessing publications that users recognise and abide by the legal requirements associated with these rights.

\section{Takedown policy}

If you believe that this document breaches copyright please refer to the University of Manchester's Takedown Procedures [http://man.ac.uk/04Y6Bo] or contact uml.scholarlycommunications@manchester.ac.uk providing relevant details, so we can investigate your claim.

\section{OPEN ACCESS}




\section{Recent Progress in MANY-BODY THEORIES VOLUMe 2}

Edited by

Y. Avishai

Ben Gurion University

Beer Sheva, Israel

Plenum Press • New York and London 
Proceedings of the Sixth International Conference on Recent Progress in Many-Body Theories, held November 5-10, 1989, in Arad, Israel

Library of Congress Catalog Card Number 88-645051

ISBN 0-306-43705-8

(C) 1990 Plenum Press, New York

A Division of Plenum Publishing Corporation

233 Spring Street, New York, N.Y. 10013

All rights reserved

No part of this book may be reproduced, stored in a retrieval system, or transmitted in any form or by any means, electronic, mechanical, photocopying, microfilming, recording, or otherwise, without written permission from the Publisher

Printed in the United States of America 


\title{
COUPLED CLUSTER PARAMETRIZATIONS OF MODEL FIELD THEORIES
}

\section{AND THEIR BARGMANN-SPACE REPRESENTATIONS}

\author{
J. S. Arponen ${ }^{*}$ and R.F. Bishop" \\ Department of Theoretical Physics, University of Helsinki \\ Siltavuorenpenger 20C, SF-00170 Helsinki, Finland \\ 'Department of Mathematics \\ University of Manchester Institute of Science and Technology \\ P O Box 88, Manchester M60 1QD, England
}

\section{INTRODUCTION}

Coupled cluster techniques ${ }^{1-7}$ have by now been very successfully applied to numerous quantum systems of strongly-interacting particles and fields. $^{2,8-12}$ One of the key features of the whole coupled cluster method (CCM) is its ability to incorporate rather naturally and at a very fundamental level, such unifying concepts as supercoherent states, generalized many-body mean fields and generalized order parameters, and exact mappings onto corresponding multilocal classical field theories. This is particularly true of the most recent version of the theory, the so-called extended coupled cluster method (ECCM). ${ }^{4,6,7}$ In common with its predecessor, the normal coupled cluster method (NCCM) of Coester and Kümmel, ${ }^{1}$ the essence of the formalism is its intrinsic universality in being able to be applied to any system governed by some underlying Schrödinger dynamics. Furthermore, the methods are both exact in principle, and capable of being systematically implemented at various levels of approximation in practice. In its most general form the CCM provides a complete dynamical description of a many-body system by formulating it in terms of a dynamical variational principle for the action. $^{4,7}$

The relationships between the ECCM, the NCCM and the more primitive configuration-interaction (CI) method, ${ }^{13}$ and the way that they form a very natural hierarchy of formulations, have been explored in some detail elsewhere. ${ }^{7} \quad$ By focusing on the interpretation of each method in terms of time-independent perturbation theory, and their decompositions in terms of Goldstone diagrams, it was possible to formulate each of the three methods in terms of suitably-defined generalized tree diagrams. Their connectivity properties are intimately related to the linked-cluster properties of the different cluster or correlation amplitudes which characterize each of the three methods. Furthermore, these linked-cluster properties are important with regard to the problem of the size-extensivity or size-consistency (i.e, full separability) of the resulting many-body description. ${ }^{14}$ Whereas the CI method suffers from well-known deficiencies in this respect, coupled cluster methods in principle take good care of these separability problems, insofar as the relevant correlation amplitudes which describe the interacting many-body system and its properties, obey the cluster property. 
In particular, in the ECCM (and only in the ECCM) all of the corresponding amplitudes which fully characterize (for example, the ground state of) the many-body system, obey the exact cluster property. Since the ECCM is therefore capable, in principle, of describing such phenomena as phase transitions, spontaneous symmetry breaking, and states of topological excitation, as well as nonequilibrium properties, its potential range of applicability is very wide. Particular applications to date include gauge-field descriptions of both a charged impurity in a polarizable medium (e.g., positron annihilation. in metals), ${ }^{5}$ and the zero-temperature quantum hydrodynamics of a strongly-interacting condensed Bose fluid. ${ }^{11}$ In view of its promise for further use, it seems particularly appropriate to investigate as rigorously as possible such fundamental features of the ECCM as the existence and convergence properties of the method at its various levels of approximation. To this end, we compare in this paper all three parametrizations (viz., $\mathrm{CI}, \mathrm{NCCM}$, and ECCM), via their holomorphic representations in the Bargmann Hilbert space. ${ }^{16}$ Each method is illustrated by applying it to various quantum anharmonic oscillators, chosen both as an important class of model field theories in themselves, and as an especially stringent test of the ECCM in particular.

One of the most important outcomes of the present work is that by utilizing the holomorphic representation of the various amplitudes, we are able to algebraize the various formulations completely. In particular the topological linking or connectivity requirements associated with the structure of the vertices in the emergent tree-diagram structures, which are normally analyzed diagrammatically, now have considerable new light shed upon them. Indeed, we know of no other comparable example where the asymptotic analytic behaviour of the various amplitudes which fully and exactly parametrize the system, has been so fully analyzed. As an example, we show how certain formally divergent series may be given precise - but generally non-unique interpretations. We stress from the outset that our final results, which take the form of a generating function for the expectation values of arbitrary operators, are rather general and not simply restricted to the illustrative case of the anharmonic oscillators.

The outline of the remainder of the paper is as follows. Sections 2 and 3 are devoted to describing the basic elements of respectively the three fundamental methods (Cl, NCCM and ECCM) and their parametrizations of the wavefunction, and the Bargmann Hilbert space and the associated coherent states which underpin the holomorphic representation. The Schrödinger and Fock representations of the wavefunction and their inter-relationships are described in Sec. 4, where we also discuss the holomorphic representation of all three ( $\mathrm{CI}$ and $\mathrm{CCM}$ ) parametrizations for the case of simple field theories with a single bosonic mode. These techniques are illustrated in more detail in Sec. 5 by specific reference to the anharmonic oscillator problem. After describing in Sec. 6 how general expectation values of arbitrary operators may be generated wholly algebraically, the results are discussed in Sec. 7 .

\section{BASIC ELEMENTS; PARAMETRIZATIONS OF THE WAVEFUNCTION}

We consider the bra and ket ground-state wavefunctions $\langle\tilde{\psi}|$ and $|\psi\rangle$ respectively, of an arbitrary many-body system,

$$
\mathrm{H}|\psi\rangle=\mathrm{E}_{0}|\psi\rangle,\langle\tilde{\psi}| \mathrm{H}=\mathrm{E}_{0}\langle\tilde{\psi}|,
$$

where we do not necessarily assume that the Hamiltonian $\mathrm{H}$ is hermitian. For ease of presentation we restrict ourselves to "closed-shell systems" for which each of the parametrizations that we consider may be referred to some suitable single model or reference state $|0\rangle$. This state can be chosen rather freely, so long as it is a cyclic vector in the sense that the algebra of all possible 
operators in the many-body Hilbert space is spanned by the two Abelian sub-algebras of creation and destruction operators defined with respect to it. In this way we may define suitable complete sets of (multiconfigurational) orthonormal creation operators $\left\{\mathrm{C}_{\mathbf{k}}^{\dagger}\right\}$, and their hermitian adjoint destruction counterparts $\left\{\mathrm{C}_{\mathrm{k}}\right\}$, where each many-body configuration-space index $\mathrm{k}$ represents some appropriate set of discrete or continuous (single-particle) labels. Their choice depends upon the specific system under consideration. Examples have been given elsewhere.

The three particular parametrizations of the ground-state wavef unction, namely the $\mathrm{CI}$ method, the NCCM, and the ECCM, are now specified respectively as follows:

CI

$$
|\psi\rangle \equiv F|0\rangle,\langle\tilde{\psi}| \equiv\langle 0| \tilde{F}
$$

$$
\mathrm{F} \equiv \sum_{\mathrm{k}} \mathrm{F}_{\mathrm{k}} \mathrm{C}_{\mathrm{k}}^{\dagger}, \tilde{\mathrm{F}} \equiv \sum_{\mathrm{k}} \tilde{\mathrm{F}}_{\mathrm{k}} \mathrm{C}_{\mathrm{k}}
$$

$$
|\psi\rangle \equiv e^{S}|0\rangle,\langle\tilde{\psi}| \equiv\langle 0| \Omega e^{-S}
$$

\section{NCCM}

ECCM

$$
\begin{aligned}
& \mathrm{S} \equiv \sum_{\mathrm{k}}^{\prime} \mathrm{S}_{\mathrm{k}} \mathrm{C}_{\mathrm{k}}^{\dagger}, \Omega \equiv \sum_{\mathrm{k}} \Omega_{\mathrm{k}} \mathrm{C}_{\mathrm{k}}=1+\sum_{\mathrm{k}}^{\prime} \Omega_{\mathrm{k}} \mathrm{C}_{\mathrm{k}} ; \\
& |\psi\rangle \equiv \mathrm{e}^{\mathrm{S}}|0\rangle,\langle\tilde{\psi}| \equiv\langle 0| \mathrm{e}^{\tilde{\Sigma}} \mathrm{e}^{-\mathrm{S}},
\end{aligned}
$$

$$
\tilde{\Sigma} \equiv \sum_{\mathrm{k}}^{\prime} \tilde{\sigma}_{\mathrm{k}} \mathrm{C}_{\mathbf{k}}
$$

where a prime on a sum over the set-index $\mathrm{k}$ indicates that the term $\mathrm{k}=0$ is excluded, where, by definition $\mathrm{C}_{0}^{\dagger} \equiv \mathrm{I}$, the identity operator. The three methods are then completely specified by the respective pairs of creation and destruction operators $\{\mathrm{F}, \widetilde{\mathrm{F}}\},\{\mathrm{S}, \Omega\}$, and $\{\Sigma, \widetilde{\Sigma}\}$, where in the latter (ECCM) case,

$$
\Sigma|0\rangle \equiv(I-|0\rangle\langle 0|) \mathrm{e}^{\tilde{\Sigma}} \mathrm{S}|0\rangle, \Sigma \equiv \sum_{\mathrm{k}}^{\prime} \sigma_{\mathrm{k}} \mathrm{C}_{\mathrm{k}}^{\dagger},
$$

or, equivalently, by the complete sets of correlation amplitudes $\left\{F_{k}, \tilde{F}_{k}\right\}$, $\left\{S_{k}, \Omega_{k}\right\}$, and $\left\{\sigma_{k}, \tilde{\sigma}_{k}\right\}$, in terms of which they may be decomposed.

If we denote these three sets of parameters generically as $\left\{\mathrm{x}_{\mathrm{k}}, \mathrm{y}_{\mathrm{k}}\right\}$, we have shown elsewhere ${ }^{7}$ that in each case the exact ground-state energy expectation value, $\overline{\mathrm{H}} \equiv \overline{\mathrm{H}}\left[\mathrm{x}_{\mathrm{k}}, \mathrm{y}_{\mathrm{k}}\right] \equiv\langle\tilde{\psi}|\mathrm{H}| \psi\rangle /\langle\tilde{\psi} \mid \psi\rangle$, is stationary with respect to each member of the set,

$$
\partial \overline{\mathrm{H}} / \partial \mathrm{x}_{\mathrm{k}}=0=\partial \overline{\mathrm{H}} / \partial \mathrm{y}_{\mathrm{k}} \text {. }
$$

These coupled sets of equations (4) determine the ground-state equilibrium values of the respective amplitudes. Only in the case of the CI method are the resulting equations linear. Both CCM parametrizations provide intrinsically nonlinear decompositions of the underlying time-independent Schrödinger equation (1). Furthermore, in a full dynamical description via 
the time-dependent Schrödinger equation, in which the energy eigenstates $|\psi\rangle$ and $\langle\widetilde{\psi}|$ are replaced by their non-stationary counterparts, the equations of motion in all three parametrizations have been shown to have the identical form,

$$
\mathrm{idx}_{\mathrm{k}} / \mathrm{dt}=\partial \overline{\mathrm{H}} / \partial \mathrm{y}_{\mathrm{k}},-\mathrm{idy} \mathrm{k}_{\mathrm{k}} / \mathrm{dt}=\partial \overline{\mathrm{H}} / \partial \mathrm{x}_{\mathrm{k}}
$$

These are just the canonical equations of motion in a classical (multi-body) phase space, in which the role of canonical co-ordinates is thus played by the multiconfigurational correlation amplitudes themselves.

The primary difference between the three parametrizations lies in their separability properties. Whereas the exact wave operators $F$ and $\tilde{F}$, and the correlation operator $\Omega$ are multiplicatively separable, the various cluster operators $S, \Sigma$, and $\tilde{\Sigma}$ are additively separable. The important physical properties of the system are extensive quantities, and these are clearly most directly formulated in terms of parameters which are additively separable even when the full description is approximated by truncation in the multiconfigurational phase space. Furthermore, this additive separability is intrinsically related to the connectivity properties of the associated amplitudes. For example, the coefficients $\left\{\mathrm{S}_{\mathrm{k}}\right\}$ are just the Hubbard linked-cluster amplitudes which represent the sums of complete sets of connected (or linked) open-ended Goldstone diagrams for the ground state, whereas the comparable coefficients $\left\{\mathrm{F}_{\mathrm{k}}\right\}$ also contain disconnected (or unlinked) terms. In fact, all of the amplitudes $\left\{\mathrm{S}_{\mathrm{k}}\right\},\left\{\sigma_{\mathrm{k}}\right\}$ and $\left\{\tilde{\sigma}_{\mathrm{k}}\right\}$ are linked, and hence obey the cluster property, whereas the amplitudes $\left\{\mathrm{F}_{\mathrm{k}}\right\},\left\{\tilde{\mathrm{F}}_{\mathrm{k}}\right\}$ and $\left\{\Omega_{\mathrm{k}}\right\}$ contain unlinked pieces. We see, in particular, that only the ECCM thus comprises a parametrization in which all of the amplitudes are linked.

The classical phase space for all three parametrizations is in principle of equal complexity. Nevertheless, the increasing amount of connectivity in the chain CI $\rightarrow$ NCCM $\rightarrow$ ECCM (which, incidentally, is also related to the various "generalized time-ordering" properties, 4 associated with the tree-diagram structures which emerge from each method in terms of their time-independent perturbation-theoretic content), is expected to bring about a similar increase in the effective compactification of the respective phase space. In particular, the ECCM phase space is a complex differentiable manifold with a symplectic structure induced by a generalized classical Poisson bracket form between the amplitudes, ${ }^{6}$ each of which is now multilocal in the sense of obeying the cluster property in co-ordinate space. The associated compactification of the phase space may be expected to allow the physically important region to be described in terms of an effective classical mean (multilocal) field theory of much reduced dimensionality and, hence, complexity.

The above discussion opens the intriguing possibility that a further exploration of the various parametrizations, and the ECCM in particular, might shed new light on the relationships between classical and quantum mechanics and on the quantization of classical systems. Despite the obvious intuitive physical appeal of the ECCM, many open questions remain. Thus, the very existence of the ECCM phase space is far from clear for infinite Hilbert spaces. The related practical problems of the convergence properties of the method (and the associated need for appropriate regularization procedures), and how to devise useful approximation schemes, are also of fundamental importance.

In order to address these issues we examine here the one-body anharmonic oscillator as a much-studied system with infinite-dimensional Hilbert space. 
As a model interacting field theory, the anharmonic oscillator is highly singular in the sense of being maximally nonlocal. It thus provides a most stringent test for a method such as the ECCM whose linked-cluster properties suit it particularly to physical systems with normal locality and separability properties. Furthermore, it is well-known that ordinary perturbation theory fails to converge for this system, however weak the anharmonicity. ${ }^{17,18}$ Nevertheless, the Fock space associated with such one-body systems can be described in terms of (functions of) a single creation operator, $a$, and its hermitian adjoint, a.

\section{BARGMANN REPRESENTATION; COHERENT STATES}

For a one-body problem in one dimension, with Hamiltonian

$$
\mathrm{H}=\frac{1}{2} \mathrm{p}^{2}+\mathrm{V}(\mathrm{x}) \text {, }
$$

the position operator $\mathrm{x}$ and its canonically conjugate momentum operator $\mathrm{p}=$ $-\mathrm{id} / \mathrm{dx}$, are conventionally mapped into their canonical Fock-space creation and destruction operator counterparts by,

$$
x=2^{-\frac{1}{2}}\left(a^{+}+a\right), p=2^{-\frac{1}{2}} i\left(a^{+}-a\right),
$$

which obey the usual bosonic canonical commutation relation $(\mathrm{CCR}),\left[\mathrm{a}, \mathrm{a}^{\boldsymbol{\dagger}}\right]=1$. The corresponding (normalized) vacuum state $|0\rangle, a|0\rangle=0$, which now plays the role of model state or cyclic vector, is simply the ground state of the harmonic oscillator Hamiltonian, $\mathrm{H}^{0}=\frac{1}{2} \mathrm{p}^{2}+\frac{1}{\mathrm{t}} \mathrm{x}^{2}$. It has the usual coordinate-space representation, $\langle\mathrm{x} \mid 0\rangle \equiv \psi^{0}(\mathrm{x})=\pi^{-\frac{1}{4}} \exp \left(-\frac{1}{2} \mathrm{x}^{2}\right)$. Arbitrary ket and bra states in the Fock space can now be represented in the form,

$$
\begin{aligned}
& |g\rangle \equiv g\left(a^{*}\right)|0\rangle,\left\langle f^{*}\right| \equiv\langle 0| f(a)=\left[f^{*}\left(a^{\dagger}\right)|0\rangle\right]^{\dagger}=\left(\left|f^{*}\right\rangle\right)^{\dagger} ; \\
& g(z) \equiv \sum_{n=0}^{\infty} g_{n} z^{n}, f(z)=\sum_{n=0}^{\infty} f_{n} z^{n} .
\end{aligned}
$$

The normalized eigenstates of the operator a are the usual Glauber coherent states $|z\rangle$,

$$
a|z\rangle=z|z\rangle ;|z\rangle=e^{\left(z a^{\dagger}-z^{*} a\right)}|0\rangle=e^{-\frac{1}{2}|z|^{2}} e^{z a^{\dagger}}|0\rangle,
$$

where $z$ is an arbitrary complex number. Their co-ordinate-space representation is readily seen to be given as,

$$
\langle x \mid z\rangle=\pi^{-\frac{1}{4}} \exp \left(-\frac{1}{2}|z|^{2}-\frac{1}{2} z^{2}+\sqrt{2} x z-\frac{1}{2} x^{2}\right) .
$$

They obey the well-known completeness and overlap relations,

$$
\begin{aligned}
& I=\pi^{-1} \int d^{2} z|z\rangle\left\langle z\left|=\int_{-\infty}^{\infty} d x\right| x\right\rangle\langle x|, \\
& \left\langle z \mid z^{\prime}\right\rangle=\exp \left(-\frac{1}{2}|z|^{2}-\frac{1}{2}\left|z^{\prime}\right|^{2}+z^{*} z^{\prime}\right) .
\end{aligned}
$$

In the Bargmann space the CCR algebra is represented by the algebra,

$$
a^{\dagger} \rightarrow z, a \rightarrow d / d z
$$

of the complex variable $z$ and its derivative. Arbitrary states $f(a) g\left(a^{*}\right)|0\rangle$ in the Fock space now have the holomorphic Bargmann representation 
$f(d / d z) g(z)$. In this way the bosonic quantum field theory in a Hilbert space may be mapped into the corresponding (classical) field theory of a complex function in a particular normed space, the so-called Bargmann Hilbert space. ${ }^{16}$ Thus, the ground-state Schrödinger equation (1) with the Hamiltonian of Eq. (6) - becomes the following ordinary differential equation in the complex plane,

$$
-\frac{1}{4}(d / d z-z)^{2} F(z)+V[(d / d z+z) / \sqrt{2}] F(z)=E_{0} F(z)
$$

in terms of the CI representation $|\psi\rangle=F\left(a^{\dagger}\right)|0\rangle$ of the ground-state wavefunction.

It is easy to check, using the CCR and Eqs. (9) and (11), that the scalar product of two such arbitrary states as in Eq.(8) can be expressed in any of the following forms,

$$
\begin{aligned}
\left\langle f^{*} \mid g\right\rangle & =\pi^{-1} \int d^{2} z e^{-|z|^{2}} f(z) g\left(z^{*}\right)=\pi^{-1} \int d^{2} z e^{-|z|^{2}} f\left(z^{*}\right) g(z) \\
& =\left.f(d / d z) g(z)\right|_{z=0} \\
& =\sum_{n=0}^{\infty} n ! f_{n} g_{n} .
\end{aligned}
$$

If the two states are normalizable, both (holomorphic) functions $f(z)$ and $g(z)$ will be entire functions of order $\rho \leq 2$, and each of the relations (14a) (14c) yields the same (convergent) result. Differences will arise only when the Hilbert space of normalizable wavefunctions is extended to a more general linear vector space which may encompass states that are not normalizable in the standard metrics. It is just such an extension which is necessitated by the CCM parametrizations of the state vectors.

\section{HOLOMORPHIC REPRESENTATIONS OF THE CI AND CCM PARAMETRIZATIONS FOR SIMPLE BOSONIC FIELD THEORIES}

In the case of simple bosonic field theories with a single mode, created by the operator $a^{f}$, we may therefore now use the Bargmann-space concepts of Sec. 3 to give a holomorphic representation of the various operators defined generally in Eqs. (2a) - (2c) that define our three basic (CI, NCCM and ECCM) parametrizations of Sec. 2. In the first place we consider the detailed mapping between the Schrödinger and Bargmann representations of an arbitrary wavef unction,

$$
|\psi\rangle=F\left(a^{\dagger}\right)|0\rangle, F(z) \equiv \sum_{n=0}^{\infty} F_{n} z^{n},
$$

written in its CI form [c.f., Eq. (2a)]. By considering the overlap $\left\langle z^{*} \mid \psi\right\rangle$, inserting a complete set of position eigenstates $|x\rangle$, and using Eq. (10), it is straightforward to derive the Fourier-like mapping,

$$
F(z)=\pi^{-\frac{1}{4}} \exp \left(-\frac{1}{2} z^{2}\right) \int_{-\infty}^{\infty} d x \exp \left(\sqrt{2} z x-\frac{1}{2} x^{2}\right) \psi(x) .
$$

By considering the Fourier transform of Eq. (17), or by likewise considering the overlap $\langle x \mid \psi\rangle$ and inserting a complete set of coherent states as in Eq. (11), one may also derive the inverse relations, 


$$
\begin{aligned}
\psi(x) & =2^{-\frac{1}{2} \pi^{-\frac{3}{4}}} \exp \left(\frac{1}{2} x^{2}\right) \int_{\infty}^{\infty} d \eta \exp \left(-i \sqrt{2} x \eta-\frac{1}{2} \eta^{2}\right) F(i \eta) \\
& =\pi^{-5 / 4} \exp \left(-\frac{1}{2} x^{2}\right) \int d^{2} z \exp \left(-|z|^{2}-\frac{1}{2} z^{* 2}+\sqrt{2} x z^{*}\right) F(z)
\end{aligned}
$$

For the further CCM parametrizations it is conventional to impose the so-called intermediate normalization condition on $|\psi\rangle$, namely $\langle 0 \mid \psi\rangle=$ $\int \mathrm{dx} \psi^{0}(\mathrm{x}) \psi(\mathrm{x})=1$, which implies that $\mathrm{F}_{0}=1$ in Eq. (16). Furthermore, the CCM representations of Eqs. (2b) - (2c) imply that $\langle\tilde{\psi} \mid \psi\rangle=1$. In the case of a hermitian Hamiltopian this simply gives $\langle\tilde{\psi}|=\mathrm{N}^{2}\langle\psi|$, where $\mathrm{N}^{2} \equiv\langle\psi \mid \psi\rangle=$ $\int \mathrm{dx}|\psi(\mathrm{x})|^{2}=\left\langle 0\left|\mathrm{~F}^{*}(\mathrm{a}) \mathrm{F}\left(\mathrm{a}^{\dagger}\right)\right| 0\right\rangle$, and hence $\tilde{\mathrm{F}}(\mathrm{a})=\mathrm{N}^{-2} \mathrm{~F}^{*}(\mathrm{a})$.

In the CCM, the wave operator $\left.\mathrm{F}^{+} \mathrm{a}^{+}\right)$is now parametrized in the exponential form,

$$
\mathrm{F}\left(\mathrm{a}^{+}\right)=\exp \mathrm{S}\left(\mathrm{a}^{+}\right) ; \mathrm{S}(\mathrm{z}) \equiv \sum_{\mathrm{n}=1}^{\infty} \mathrm{S}_{\mathrm{n}} \mathrm{z}^{\mathrm{n}}
$$

The remaining NCCM operator $\Omega(a)$, where $\langle\tilde{\psi}| \equiv\langle 0| \Omega(a) \exp \left[-\mathrm{S}\left(\mathrm{a}^{\dagger}\right)\right]$, is then simply given as,

$$
<0\left|\Omega(a)=N^{-2}<0\right| F^{*}(a) F\left(a^{\dagger}\right) ; \Omega(z) \equiv \sum_{n=0}^{\infty} \Omega_{n} z^{n},
$$

with $\Omega_{0}=1$. If we define the average value of an arbitrary operator $\supseteqq=\mathfrak{O}\left(\mathrm{a}^{\dagger}, \mathrm{a}\right)$ as $\langle\supseteqq\rangle \equiv \overline{\mathfrak{D}} \equiv\langle\tilde{\psi}|\supseteqq| \psi\rangle=\mathrm{N}^{-2}\left\langle 0\left|\mathrm{~F}^{*}(\mathrm{a}) \mathfrak{O}\left(\mathrm{a}^{\dagger}, \mathrm{a}\right) \mathrm{F}\left(\mathrm{a}^{\dagger}\right)\right| 0\right\rangle$, it is immediately clear from Eq. (20) that the function $\Omega(z)$ is the moment-generating function for the powers of the creation operator $a$,

$$
\Omega_{\mathrm{n}}=\left\langle\left(\mathrm{a}^{+}\right)^{\mathrm{n}}\right\rangle / \mathrm{n} !, \Omega(\mathrm{z})=\left\langle\exp \left(\mathrm{za}^{+}\right)\right\rangle
$$

By inserting a complete set of co-ordinate states $|x\rangle$ into the representation of Eq. (21) and making use of Eq. (7), we readily find the expression,

$$
\Omega(z)=N^{-2} \int_{-\infty}^{\infty} \mathrm{dx} \psi^{*}(\mathrm{x}) \exp \left[2^{-\frac{1}{2}} \mathrm{z}(\mathrm{x}-\mathrm{d} / \mathrm{dx})\right] \psi(\mathrm{x}) .
$$

The exponential operator is easily transformed by use of the Baker-Campbell-Hausdorff theorem to yield the explicit representation,

$$
\Omega(z)=N^{-2} \exp \left(-\frac{1}{4} z^{2}\right) \int_{-\infty}^{\infty} d x \exp (x z / \sqrt{2}) \psi^{*}(x) \psi(x-z / \sqrt{2}),
$$

which is bilinear in the Schrödinger wavefunction.

In the ECCM parametrization, the function $\tilde{\Sigma}(z)$ of Eq. (2c) is given by $\Omega(z)=\exp \tilde{\Sigma}(z)$, and is hence simply the associated cumulant function whose coefficients provide a measure of the connected averages,

$$
\tilde{\Sigma}(z) \equiv \sum_{n=1}^{\infty} \tilde{\sigma}_{n} z^{n}, \tilde{\sigma}_{n}=\frac{1}{n !}\left\langle\left(a^{+}\right)^{n}\right\rangle \text { conn. }
$$

The relationship between the coefficients $\Omega_{\mathrm{n}}$ and $\tilde{\sigma}_{\mathrm{n}}$ is thus just the usual one between the moments and cumulants of a probability distribution. Finally, the remaining ECCM function $\Sigma\left(a^{+}\right)$given by Eq. (3), is readily shown to have the form, 


$$
\Sigma(z) \equiv \sum_{n=1}^{\infty} \sigma_{n} z^{n}=\left\langle 0\left|\Omega(a)\left(e^{z a}-1\right) S\left(a^{+}\right)\right| 0\right\rangle,
$$

$$
\sigma_{\mathrm{n}}=\sum_{\mathrm{m}=0}^{\infty} \frac{(\mathrm{m}+\mathrm{n}) !}{\mathrm{n} !} \Omega_{\mathrm{m}} \mathrm{S}_{\mathrm{m}+\mathrm{n}}=\left.\frac{1}{\mathrm{n} !} \frac{\mathrm{d}^{\mathrm{n}}}{\mathrm{dz} \mathrm{d}^{\mathrm{n}}} \Omega(\mathrm{d} / \mathrm{dz}) \mathrm{S}(\mathrm{z})\right|_{\mathrm{z}=0} .
$$

If an arbitrary operator $\mathrm{A}\left(\mathrm{a}^{\dagger}, \mathrm{a}\right)$ and the functions $\exp \left[ \pm \mathrm{S}\left(\mathrm{a}^{\dagger}\right)\right]$ are now expressed in their Bargmann form, we readily find, for example, that the NCCM form of the average-value functional $\bar{A}$ has the holomorphic representation,

$$
\overline{\mathrm{A}}=\left.\Omega(\mathrm{d} / \mathrm{d} z) \mathrm{A}\left(\mathrm{z}, \mathrm{d} / \mathrm{d} \mathrm{z}+\mathrm{S}^{\prime}(\mathrm{z})\right)\right|_{\mathrm{z}=0},
$$

where $S_{+}^{\prime}(z) \equiv d S / d z$. When $|\psi\rangle$ and $\langle\tilde{\psi}|$ are the exact ground eigenstates of $\mathrm{H}=\mathrm{H}\left(\mathrm{a}^{+}, \mathrm{a}\right)$, the usual coupled set of nonlinear NCCM equations for the coefficients $\left\{\mathrm{S}_{\mathrm{n}}\right\}$,

$$
\left\langle 0\left|a^{n} e^{-S} H e^{S}\right| 0\right\rangle=0 ; n=1,2, \ldots,
$$

which follows immediately from the Schrödinger equation (1), now simply correspond to the stationarity condition [c.f., Eq. (4)],

$$
\partial \overline{\mathrm{H}} / \partial \Omega_{\mathrm{n}}=\left.(\mathrm{d} / \mathrm{d} z)^{\mathrm{n}} \mathrm{H}\left(\mathrm{z}, \mathrm{d} / \mathrm{dz}+\mathrm{S}^{\prime}(\mathrm{z})\right)\right|_{\mathrm{z}=0}=0 ; \mathrm{n} \geq 1 \text {, }
$$

in terms of the NCCM average-value functional $\bar{H}=\bar{H}\left(S_{n}, \Omega_{n}\right)$. Similarly, the remaining NCCM equations for the coefficients $\left\{\Omega_{n}\right\}$,

$$
\left\langle 0\left|\Omega \mathrm{e}^{-\mathrm{S}}\left[\mathrm{H},\left(\mathrm{a}^{\dagger}\right)^{\mathrm{n}}\right] \mathrm{e}^{\mathrm{S}}\right| \mathrm{O}\right\rangle=0 ; \mathbf{n}=1,2, \ldots,
$$

correspond to the stationarity condition,

$$
\partial \overline{\mathrm{H}} / \partial \mathrm{S}_{\mathrm{n}}=\left.\Omega(\mathrm{d} / \mathrm{d} z)\left[\mathrm{H}\left(\mathrm{z}, \mathrm{d} / \mathrm{d} z+\mathrm{S}^{\prime}(\mathrm{z})\right), \mathrm{z}^{\mathrm{n}}\right]\right|_{\mathrm{z}=0}=0 ; \mathrm{n} \geq 1 .
$$

The $\mathrm{CI}$ and ECCM stationarity equations may be comparably expressed. It is clear from the above discussion that it will be useful to study the analytic properties of the various functions $F(z), S(z), \Omega(z), \quad \Sigma(z)$ and $\tilde{\Sigma}(z)$ at the exact ground-state stationary point and in its vicinity. In order to make further progress it is simplest to treat a specific example.

\section{EXAMPLE: THE ANHARMONIC OSCILLATOR}

We now consider the anharmonic oscillator model, corresponding to Eqs. (1) and (6) with

$$
\mathrm{V}(\mathrm{x})=\frac{1}{2} \lambda \mathrm{x}^{2 \mathrm{~K}}, \mathrm{~K}=2,3, \ldots
$$

If we write $F(z) \equiv \exp \left(-\frac{1}{2} z^{2}\right) f(z)$, Eq. (14) shows that the function $f(z)$ satisfies the differential equation,

$$
\left(\frac{d}{d z}\right)^{2 K_{f}} f(z)=\frac{2^{K+1}}{\lambda}\left[\left(\frac{1}{2} \frac{d}{d z}-z\right)^{2}+E_{0}\right] f(z) .
$$

By using Eq. (17) and the fact that the ground-state wavefunction $\psi(x)$ is real, positive, and of even parity, we see easily that $f(z)$ is an even entire function of $z$ which is real and positive on the real axis. Equation (32) shows that in the asymptotic region where $r=|z| \rightarrow \infty$, there are $2 \mathrm{~K}$ 
qualitatively different solutions,

$$
\begin{aligned}
& \mathrm{f}_{(\mathrm{n})}(\mathrm{z}) \rightarrow \exp \left(\mathrm{b} \varepsilon^{\mathrm{n}} \mathrm{z}^{v}\right) ; \mathrm{n}=-\mathrm{K}+1, \ldots, \mathrm{K}, \\
& \nu \equiv \frac{\mathrm{K}+1}{\mathrm{~K}}, \varepsilon \equiv \exp \left(\frac{\mathrm{i} \pi}{\mathrm{K}}\right), \mathrm{b} \equiv \frac{1}{v} 2^{v / 2} \lambda^{-(v-1) / 2},
\end{aligned}
$$

and hence that $f(z)$ is an entire function of order $v$. The normalizability of the ground-state wavefunction $\psi(x)$ implies from Eqs. (15a) and (16) that the product $\exp \left(-|z|^{2}\right)|\mathrm{F}(z)|^{2}$ is integrable over the whole complex plane. Hence, for real $x$ and $y$, we have $|f(x+i y)| \rightarrow o(1)$ as $y \rightarrow \pm \infty$. Thus, for example, in the first quadrant, $0 \leq \arg (z)<\frac{1}{2} \pi$, of the complex plane, only the $\mathrm{K}$ asymptotic branches of Eq. (33) with $\mathrm{n}=0,1, \ldots, \mathrm{K}-1$ are permitted, and in the fourth quadrant, $-\frac{1}{2} \pi<\arg (z) \leq 0$, only the $K$ branches with $n=0,-1, \ldots$, $-\mathrm{K}+1$. By matching the various branches, using the usual WKB arguments, one can show that the actual asymptotic behaviour is given by,

$$
f(z) \underset{r \rightarrow \infty}{\longrightarrow} A \exp \left[b( \pm z)^{\nu}\right],|\arg ( \pm z)|<\frac{1}{2} \pi .
$$

In order to investigate the analytic behaviour of the CCM function $S(z)=$ In $F(z)$, we are also interested in the zeros $z_{m}$ of $F(z), F\left(z_{m}\right)=0$. Similar WKB arguments to those used above show that on the imaginary axis the asymptotic solutions of Eq. (34) in the left and right half-planes are of equal magnitude and can generate an infinite sequence of zeros on the imaginary axis, with asymptotic positions at $z_{m}= \pm i y_{m}$, where

$$
\mathrm{y}_{\mathrm{m}} \underset{\mathrm{m} \rightarrow \infty}{\longrightarrow}\left[\frac{\pi}{\mathrm{b}} \sec \left(\frac{\pi}{2 \mathrm{~K}}\right)\right]^{1 / \nu_{\mathrm{m}}}{ }^{1 / \nu}
$$

It also seems probable that all of the zeros lie on the imaginary axis.

In order to examine the analytic properties of the NCCM function $\Omega(z)$, we may use the representation of Eq. (23). It is easy to show that $\psi(x)$ can be analytically continued into the complex plane where it is an entire function of order $\mathrm{K}+1$. One may then use its asymptotic behaviour in Eq. (23) to show that $\Omega(z)$ is also an entire function of order $K+1$, with asymptotic behaviour $\Omega(x) \sim \exp \left(-k \sqrt{\lambda}|x|^{k+1}\right)$, where $k$ is a constant, as $|x| \rightarrow \infty$. Equation (23) also shows that $\Omega(z)$ has no zeros on the real axis.

We may now use the Hadamard decomposition theorem and the fact that $f(z)$ is an entire function of order $1<v<2$, to yield the representations,

$$
f(z)=\prod_{m}\left(1-z / z_{m}\right) \exp \left(z / z_{m}\right), s(z)=-\frac{1}{2} z^{2}+\sum_{m}\left[\ln \left(1-z / z_{m}\right)+z / z_{m}\right],
$$

where the product and sum run over the infinite sequence $\left\{z_{\mathrm{m}}\right\}$ of zeros of $f(z)$. It is now immediately clear, for example, that the state $S\left(a^{+}\right)|0\rangle$ is not normalizable within the Hilbert space. One consequence is that the CCM operator $\mathrm{S}(\mathrm{a})$ cannot simply be reckoned to be an operator which generates "small" correlations in some suitably defined perturbative sense, even when one might naively intuit that this is possible (e.g., in the case of small coupling constants, $\lambda$ ). By comparing Eqs. (19) and (36), and recalling that $f(z)=f(-z)$, and hence that $S(z)=S(-z)$, we find

$$
\mathrm{S}_{2 \mathrm{n}}=-\frac{1}{2} \delta_{\mathrm{n}, 1}-\frac{1}{2 \mathrm{n}} \sum_{\mathrm{m}} z_{\mathrm{m}}^{-2 \mathrm{n}}, \mathrm{n} \geq 1
$$

Equation (35) shows that the sum in Eq. (37) is convergent for all $n \geq 1$, and that the coefficients for high $\mathrm{n}$ are determined only by the two zeros, $\pm z_{1}$ 
say, closest to the origin. Thus, if $z_{1}=i \rho$, the sequence becomes asymptotically geometric,

$$
S_{2 n} \underset{n \rightarrow \infty}{\longrightarrow} \frac{(-1)^{n-1}}{n} \rho^{-2 n} .
$$

Turning now to the ECCM function $\Sigma(z)$, another consequence of the non-normalizability of the function $\mathrm{S}\left(\mathrm{a}^{+}\right)|0\rangle$ is that formal expressions such as those in Eq. (25) must be handled with extreme caution. For example, the infinite sum for $\sigma_{n}$ is easily seen to be formally divergent. Among the several techniques that may be applied to give an interpretation to these divergent or ill-defined (non-unique) expressions, we mention that Fourier and Borel resummation methods may respectively be applied to the definitions (15b) and $(15 \mathrm{c})$ of the general scalar product of the arbitrary functions $\left\langle\mathrm{f}^{*}\right|$ and $|g\rangle$ of Eq. (8). For example, in the latter case we may write

$$
\left\langle 0\left|f(a) g\left(a^{\dagger}\right)\right| 0\right\rangle=\int_{0}^{\infty . \eta} d w e^{-w}\left\langle 0\left|f(a) g_{B}\left(w^{\dagger}\right)\right| 0\right\rangle,
$$

where the integration variable $\mathrm{w}$ is in the direction of the unit vector $\eta \equiv$ $\exp (i \varphi)$ with $|\varphi|<\pi / 2$, and where

$$
g_{B}(z) \equiv \sum_{n=0}^{\infty} \frac{1}{n !} g_{n} z^{n}
$$

is the Borel transform of $g(z)$ given by Eq. (8).

Such techniques may now be employed to evaluate the ECCM function $\Sigma(z)$, calculated in terms of its first derivative from Eq. (25) as,

$$
\Sigma^{\prime}(\mathrm{z})=\left\langle 0\left|\Omega(\mathrm{a}) \mathrm{e}^{\mathrm{za}} \mathrm{S}^{\prime}\left(\mathrm{a}^{\dagger}\right)\right| 0\right\rangle=\left.\Omega(\mathrm{d} / \mathrm{du}) \mathrm{S}^{\prime}(\mathrm{u}+\mathrm{z})\right|_{\mathrm{u}=0},
$$

in which the multi-valued function $S(z)$ has been replaced by its single-valued first derivative $S^{\prime}(z)$. Thus, given that the Borel transform of the function $g(z)=1 /(z-c)$ is $g_{B}(z)=-c^{-1} \exp (z / c)$, and that for an arbitrary entire function $f(z)$ one has the simple relation,

$$
\mathrm{f}(\mathrm{a}) \exp \left(\xi \mathrm{a}^{\dagger}\right)=\exp \left(\xi \mathrm{a}^{\dagger}\right) \mathrm{f}(\mathrm{a}+\xi) \text {, }
$$

where $\xi$ is an arbitrary constant, we find the important relation,

$$
\left\langle 0\left|\mathrm{f}(\mathrm{a}) \frac{1}{\left(\mathrm{a}^{+}-\mathrm{c}\right)}\right| 0\right\rangle=-\frac{1}{\mathrm{c}} \int_{0}^{\infty \cdot \eta} \mathrm{dw} \mathrm{e}^{-\mathrm{w}_{\mathrm{f}}(\mathrm{w} / \mathrm{c})},
$$

for a class of functions $f(z)$. The direction of integration must be chosen so that the integral converges. Within the half-plane $|\arg (\eta)|<\pi / 2$ there may be several disjoint sectors in which this occurs, in which case the scalar product would not be uniquely defined.

We may now apply Eq. (43) to Eq. (41), with $S(z)$ given by the representation of Eq. (36). The asymptotic behaviour of the entire function $\Omega(z)$ and the distribution of the poles of $S^{\prime}(z)$ [and see Eq. (35)] together imply that the function $\Sigma(z)$ does not have a unique representation. Upon expansion, each such solution does however lead to precisely the same (divergent) sum of Eq. (25). The existence of such a multiplicity of solutions must ultimately be caused by the nonlinear CCM decomposition of the original linear Schrödinger eigenvalue problem. On the other hand, it is still possible to impose such physical constraints as that the ground-state 
wavefunction $\psi(\mathrm{x})$ be real and of even parity (which imply in turn that in Eq. (25) the coefficients $\sigma_{n}$ are both real and non-zero only for $n$ even), in order to construct a unique $\Sigma(z)$.

We omit the somewhat involved details of the remaining derivation and quote only the result, in terms of the odd function $R(x)$ defined, for $x \in \mathbb{R}$, as

$$
R(x) \equiv \delta^{\prime}(x)-\frac{1}{2} \operatorname{sgn}(x) \sum_{m} \exp \left(-x z_{m}\right)
$$

where $\delta(x)$ is the Dirac delta. From the asymptotic distribution of the zeros $\left\{z_{\mathrm{m}}\right\}$ of $F(z)$ from Eq. (35), it is clear that $R(x)$ is to be understood as a generalized function or distribution, in the usual Schwartz sense. Thus, the sum in Eq. (44) is formally divergent and needs to be interpreted. The sum is first partitioned into terms which are analytic in either the upper or lower complex $\mathrm{x}$-plane, and the function $\mathrm{R}(\mathrm{x})$ is then defined in terms of the boundary values of these sectionally holomorphic functions on the real axis. In this way, we derive our final expressions for $S(z)$ and $\Sigma(z)$ in terms of their first derivatives as,

$$
S^{\prime}(z)=\int_{-\infty}^{\infty} d x R(x) \sinh (x z), \Sigma^{\prime}(z)=\int_{-\infty}^{\infty} d x p(x) \sinh (x z)
$$

where $\rho(x) \equiv R(x) \Omega(x)$. Equivalently, we have that $S(z)$ and $\Sigma(z)$ are even functions with non-vanishing coefficients,

$$
S_{2 n}=\frac{1}{(2 n) !} \int_{-\infty}^{\infty} d x R(x) x^{2 n-1}, \sigma_{2 n}=\frac{1}{(2 n) !} \int_{-\infty}^{\infty} d x \rho(x) x^{2 n-1},
$$

given in terms of the moments of the respective distributions $\mathrm{R}(\mathrm{x})$ and $\rho(\mathrm{x})$. It is clear that whereas $\Sigma(z)$ is defined via Eq. (45) as an entire function, $\mathrm{S}(z)$ is only similarly defined in an infinitesimal region around the origin and on the imaginary axis, from where it may, however, be analytically continued.

\section{ARBITRARY EXPECTATION VALUES: A GENERATING FUNCTION}

We turn finally to the question of calculating the exact ground-state expectation value of an arbitrary operator in terms of either set of complete CCM coefficients, namely $\left\{\Omega_{n}, S_{n}\right\}$ for the NCCM and $\left\{\sigma_{n}, \tilde{\sigma}_{n}\right\}$ for the ECCM. To be

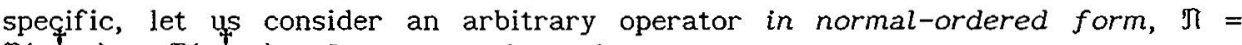
$\pi\left(a^{\dagger}, a\right) \equiv: \pi\left(a^{\top}, a\right):$. Its expectation value,

$$
\overrightarrow{\mathfrak{N}} \equiv\left\langle\Re\left(\mathrm{a}^{+}, \mathrm{a}\right)\right\rangle=\left\langle 0\left|\Omega(\mathrm{a}) \mathrm{e}^{-\mathrm{S}\left(\mathrm{a}^{\dagger}\right)} \mathfrak{N}\left(\mathrm{a}^{+}, \mathrm{a}\right) \mathrm{e}^{\mathrm{S}\left(\mathrm{a}^{\dagger}\right)}\right| 0\right\rangle,
$$

may then be given in terms of a generating function $A(u, v)$ as follows,

$$
\bar{\Re}=\left.\mathfrak{N}\left(\frac{\partial}{\partial u}, \frac{\partial}{\partial v}\right) A(u, v)\right|_{u=v=0} ; A(u, v) \equiv\left\langle e^{u a^{\dagger}} e^{v a}\right\rangle \text {. }
$$

By making use of Eq. (42), we readily derive the relation,

$$
A(u, v) \equiv\left\langle 0\left|\Omega(a+u) \exp \left[\int_{0}^{v} d w S^{\prime}(a+w)\right]\right| 0\right\rangle \text {. }
$$

By expanding the exponential as a power series, by using the explicit representation of Eq. (45), and by using the relation (42), it is then a 
simple matter to prove the respective NCCM and ECCM parametrizations of the expectation-value generating function,

$$
\begin{aligned}
A(u, v)= & \sum_{n=0}^{\infty} \frac{1}{n !} \prod_{i=1}^{n}\left[\int_{-\infty}^{\infty} d x_{i} R\left(x_{i}\right)\left(\frac{e^{v x_{i}}-1}{x_{i}}\right)\right] \Omega\left(u+x_{1}+\ldots+x_{n}\right), \\
= & \sum_{n=0}^{\infty} \frac{1}{n !} \prod_{i=1}^{n}\left[\int_{-\infty}^{\infty} d x_{i} \rho\left(x_{i}\right)\left(\frac{e^{v x_{i}}-1}{x_{i}}\right)\right] \\
& \times \exp \left[\tilde{\Sigma}\left(u+x_{1}+\ldots+x_{n}\right)-\tilde{\Sigma}\left(x_{1}\right)-\ldots-\tilde{\Sigma}\left(x_{n}\right)\right] .
\end{aligned}
$$

By using the expansions (20) and (24) together with Eq. (46), it is clear that Eqs. (48) and (50) now permit an expansion of the average-value functional in either the NCCM form $\bar{\Re}=\overline{\mathfrak{N}}\left(S_{n}, \Omega_{n}\right)$ or the ECCM form $\bar{\Re}=\bar{\Re}\left(\sigma_{n}, \tilde{\sigma}_{n}\right)$. In both cases we have infinite-order multinomials of the respective sets of coefficients. On the other hand Eqs. (50a) and (50b) represent closed-form expressions for $\bar{\pi}$ as a functional of only two functions, namely $\bar{\pi}=$ $\overline{\hat{N}}[R(x), \Omega(x)]$ for the NCCM and $\overline{\mathfrak{N}}=\overline{\mathfrak{N}}[\rho(\mathrm{x}), \tilde{\Sigma}(\mathrm{x})]$ for the ECCM. For some proper classes of these two pairs of functions, the integral expressions from Eqs. (48) and (50) are clearly convergent, whereas by contrast the convergence properties of the expansions in terms of the respective coefficient are far from clear. On the other hand, these expansions may be given a diagrammatic representation in the usual way, and it is clear that their precise linking and connectivity properties are a direct reflection of the more well-known linked (L) and double-linked (DL) expansions for the NCCM and ECCM average-value functionals of an arbitrary operator $\supseteq$, that have been described more fully elsewhere, ${ }^{2,4,6}$ and which may be given in the respective operator forms,

$$
\begin{aligned}
& \bar{\jmath}=\sum_{n=0}^{\infty} \frac{1}{n !}\left\langle 0\left|\Omega\left(\supseteqq S^{n}\right)_{L}\right| 0\right\rangle \\
& =\sum_{n=0}^{\infty} \frac{1}{n !}\left\langle 0\left|e^{\tilde{\Sigma}}(\supseteqq \Sigma)_{L}^{n}\right| 0\right\rangle_{D L} .
\end{aligned}
$$

As a specific illustration we consider, for example, the terms proportional to $\sigma_{2} \sigma_{4} \tilde{\sigma}_{2} \tilde{\sigma}_{4}$ in the ECCM expansion of the expectation value $\left\langle\left(\mathrm{a}^{+}\right)^{2} \mathrm{a}^{2}\right\rangle$, which we denote by $\mathcal{T}$, say. From Eq. (50) we have

$$
\begin{aligned}
& \left\langle e^{u a^{+}} a^{2}\right\rangle=\int_{-\infty}^{\infty} d x_{1} x_{1} \rho\left(x_{1}\right) e^{\tilde{\Sigma}\left(u+x_{1}\right)-\tilde{\Sigma}\left(x_{1}\right)} \\
& \quad+\int_{-\infty}^{\infty} d x_{1} \int_{-\infty}^{\infty} d x_{2} \rho\left(x_{1}\right) \rho\left(x_{2}\right) e^{\tilde{\Sigma}\left(u+x_{1}+x_{2}\right)-\tilde{\Sigma}\left(x_{1}\right)-\tilde{\Sigma}\left(x_{2}\right)} .
\end{aligned}
$$

Obviously the terms $\mathcal{T}$ arise from the second term in Eq. (52). By expanding out the exponential in the integrand, and keeping terms of order $\mathrm{u}^{2}$, we find that the terms $\mathcal{T}$ of interest arise from the term,

$$
\begin{aligned}
\left\langle\left(\mathrm{a}^{+}\right)^{2} \mathrm{a}^{2}\right\rangle_{\mathrm{g}},= & 2 \tilde{\sigma}_{2} \tilde{\sigma}_{4} \int_{-\infty}^{\infty} \mathrm{dx} \int_{1} \int_{\infty}^{\infty} \mathrm{dx} \mathrm{x}_{2} \rho\left(\mathrm{x}_{1}\right) \rho\left(\mathrm{x}_{2}\right)\left[\left\{\left(\mathrm{x}_{1}+\mathrm{x}_{2}\right)^{4}-\mathrm{x}_{1}^{4}-\mathrm{x}_{2}{ }^{4}\right\}\right. \\
& \left.+\left\{6\left(\mathrm{x}_{1}+\mathrm{x}_{2}\right)^{2}\left[\left(\mathrm{x}_{1}+\mathrm{x}_{2}\right)^{2}-\mathrm{x}_{1}{ }^{2}-\mathrm{x}_{2}{ }^{2}\right]\right\}+\left\{8\left(\mathrm{x}_{1}+\mathrm{x}_{2}\right)^{4}\right\}\right]
\end{aligned}
$$


In particular, Eq. (46) shows that the terms $\mathscr{T}$ arise from the terms in the integrand of Eq. (53) proportional to $x_{1} x_{2}$ or $x_{1} x_{2}{ }^{3}$. The three terms in braces in Eq. (53) lead to three contributions labelled (a), (b) and (c) respectively with specific numerical values $96 \mathrm{k}_{1} \sigma_{2} \sigma_{4} \tilde{\sigma}_{2} \tilde{\sigma}_{4}$, where $\mathrm{k}_{\mathrm{a}}=8$, $\mathrm{k}_{\mathrm{b}}=24, \mathrm{k}_{\mathrm{c}}=64$. Equation (53) also shows that the connectivity properties of the associated diagrams are as shown in Figs. (1a) - (1c) respectively, where the diagram (c) should also include an (unshown) "exchange contribution" to itself obtained by reconnecting the line joining $\tilde{\sigma}_{2}$ to $\sigma_{2}$ so that it connects $\tilde{\sigma}_{2}$ to $\sigma_{4}$ and simultaneously reconnecting any one of the lines joining $\tilde{\sigma}_{4}$ to $\sigma_{4}$ so that it connects $\tilde{\sigma}_{4}$ to $\sigma_{2}$.

\section{DISCUSSION}

It should be clear from the above discussion that we have succeeded in achieving a complete algebraization of the $\mathrm{CI}$ and CCM parametrizations and their various important properties, by utilising the holomorphic representations for our model field theories in their respective Bargmann Hilbert spaces. Most particularly, the abstract topological linking requirements related to the vertex structure of the corresponding tree diagrams associated with their respective expansions, have been especially illuminated. It should be emphasized that although we have illustrated the techniques by reference to the anharmonic oscillator problem, many of the final results of Secs. 5 and 6 are much more general. This is especially true of the form of the holomorphic representation of the CCM amplitudes in terms of the distribution of zeros for the CI wavefunction $F(z)$ [and see Eq. (45)], and the corresponding form of the generating function for arbitrary expectation values [and see Eq. (50)].

We have shown how the linked-cluster amplitudes $\left\{S_{n}\right\}$ and $\left\{\sigma_{n}\right\}$ may be represented as the moments of certain Schwartz distributions, which themselves depend on the distribution of the zeros of the holomorphic wavefunction $F(z)$.

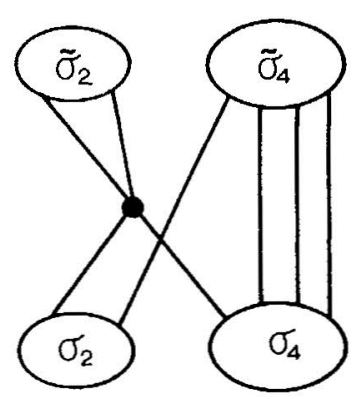

a

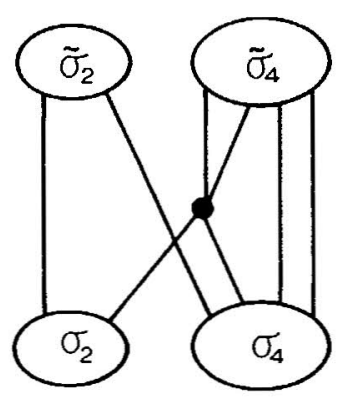

b

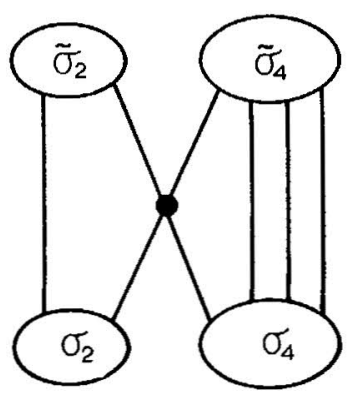

c

Fig. 1. Diagrammatic representation of some terms in the ECCM expansion of the expectation value $\left\langle\left(a^{+}\right)^{2} a^{2}\right\rangle$. 
Whereas the amplitudes $\left\{S_{n}\right\}$ decrease asymptotically as a geometric series, the amplitudes $\left\{\sigma_{\mathbf{n}}\right\}$ decrease much more rapidly in the same large-n limit. This has the consequence that their practical calculation is extremely difficult. In turn, this difference in the asymptotic behaviour of the two sets of amplitudes is itself a consequence of the fact that the ECCM function $\Sigma(z)$ is a holomorphic function, whereas its NCCM counterpart $S(z)$ is nonanalytic since it has an infinite number of branch points.

In practical implementations of the $\mathrm{CI}$ method or either version of the $\mathrm{CCM}$, one must approximate. In each case, the natural truncation scheme is the so-called $S U B(N)$ hierarchy in which the respective infinite amplitude expansions (in the appropriate configuration space) of the fundamental operators described in Eqs. (2a) - (2c), are truncated at the level where the set-index $k$ contains no more than $\mathrm{N}$ single-particle (or single pairs of particle-hole) labels. For our simple model field theories considered here, the otherwise infinite expansions of Eqs. (16), (19), (20), (24) and (25) are simply curtailed as finite $\mathrm{N}^{\text {th }}$-order polynomials. The potentially hazardous nature of these truncations is now easy to see. Thus, we know that as $|z| \rightarrow \infty$, the asymptotic behaviour of $F(z)$ is as given in Eq. (34), where $v<2$ for the anharmonic oscillator models. Nevertheless, in the NCCM representation, for example, $F(z)$ is replaced in the SUB $(N)$ approximation by a function $F_{(N)}(z)$ whose asymptotic behaviour is proportional to $\exp \left(\mathrm{S}_{\mathrm{N}} \mathrm{z}^{\mathrm{N}}\right)$ as $|z| \rightarrow \infty$. Clearly, the corresponding approximated wavefunctions $F_{(N)}\left(a^{\dagger}\right)|0\rangle$ are no longer normalizable if $\mathrm{N}>2$. Hence, such NCCM approximation schemes automatically involve excursions out of the usual Hilbert space.

Similarly, whereas the exact NCCM parametrization requires the function $S(z)$ to satisfy Eq. (28) for all $n$, namely to satisfy the condition that the function $\widetilde{H}(z) \equiv H\left(z, d / d z+S^{\prime}(z)\right)$ be a constant $\left(=E_{0}\right)$ everywhere, the $S U B(N)$

approximation merely requires that its first $N$ derivatives vanish at the origin. Thus, $\widetilde{\mathrm{H}}(z)$ is ultimately represented in the SUB(N) approximation by a finite-order polynomial, and it is clear that although the behaviour near the origin may be very well represented, the asymptotic behaviour is seriously in error. Since the physically important region is in the immediate vicinity of the origin, we may thus easily understand both why the $\operatorname{SUB}(N)$ scheme suffers from such seemingly serious drawbacks as lack of normalizability, and yet can be extremely accurate in practice, even at very low levels of the truncation index $\mathrm{N}$.

In conclusion, it is interesting to enquire how the formalism as defveloped here for simple model field theories with a single creation operator $\mathrm{a}^{+}$, might be extended to local continuum field theories where $\mathrm{a}^{+} \rightarrow \mathrm{a}^{+}(\mathrm{x})$. In such cases the complex variable $z$ must presumably be replaced by a complex field $z(x)$, and the holomorphic wavefunction $F(z)$ and its zeros by $a$ corresponding wavefunctional $F[z(x)]$ and its zero functions. Whether a practical formulation of such concepts can be realized, remains a goal for the future. In any case, we believe that the present work has already paved the way to a deeper understanding of both formulations of the CCM.

\section{ACKNOWLEDGEMENT}

One of us (RFB) gratefully acknowledges support for this work in the form of a research grant from the Science and Engineering Research Council of Great Britain. 


\section{REFERENCES}

1. $\quad$ F Coester, Nucl. Phys. $\underline{7}$ : 421 (1958);

F Coester and H G Kümmel, Nucl. Phys. 17: 477 (1960).

2. H G Kümmel, K H Lührmann and J G Zabolitzky, Phys. Rep. 36C: 1 (1978).

3. R F Bishop and K H Lührmann, Phys. Rev. B 17: 3757 (1978).

4. J Arponen, Ann. Phys. (NY) 151: 311 (1983).

5. R F Bishop and H G Kümmel, Phys. Today 40 (No. 3): 52 (1987).

6. J S Arponen, R F Bishop and E Pajanne, Phys. Rev. A 36: 2519 (1987); ibid. 2539 (1987).

7. J Arponen, R F Bishop and E Pajanne, in: "Condensed Matter Theories", Vol. 2, P Vashishta, R K Kalia and R F Bishop (eds.), Plenum, New York (1987), p. 357.

8. R F Bishop and K H Lührmann, Phys. Rev. B 26: 5523 (1982).

9. H G Kümmel, in: "Nucleon-Nucleon Interaction and Nuclear Many-Body Problems", S S Wu and T T S Kuo (eds.), World Scientific, Singapore (1984), p. 46.

10. H Kümmel, in: "Recent Progress in Many-Body Theories", Vol. 1, A J Kallio, E Pajanne and R F Bishop (eds.), Plenum, New York (1988), p. 265 .

11. J Arponen, R F Bishop, E Pajanne and N I Robinson, Phys. Rev. A 37: 1065 (1988).

12. J Noga, S A Kucharski and R J Bartlett, J. Chem. Phys. 90: 3399 (1989).

13. R K Nesbet, Phys. Rev. 109: 1632 (1958).

14. R Chowdhuri, D Mukherjee and M D Prasad, in: "Aspects of Many-Body Effects in Molecules and Extended Systems", D Mukherjee (ed.), Lecture Notes in Chemistry Vol. $\underline{50}$, Springer, Berlin (1989), p. 3.

15. J Arponen, R F Bishop and E Pajanne, in: "Condensed Matter Theories", Vol. $\underline{2}$, P Vashishta, R K Kalia and R F Bishop (eds.), Plenum, New York (1987), p. 373.

16. V Bargmann, Comm. Pure Appl. Math. 14: 180, 187, (1961); ibid. 20: 1 (1967); Rep. Math. Phys. 2.: 221 (1971).

17. C M Bender and T T S Wu, Phys. Rev. 184: 1231 (1969); Phys. Rev. D 7: 1620 (1973).

18. B Simon, Ann. Phys. (NY) 58: 76 (1970). 